\title{
Frequency of Face and Mask Contacts Before and After Quarantine During COVID-19 Pandemics
}

\author{
Mauricio Orozco Levi ${ }^{1 *}$, Diana Carolina Tiga Loza ${ }^{1,2,3}$, Mabel Reyes Pulido ${ }^{1,3}$, Alba Ramírez \\ Sarmiento ${ }^{1}$ and Victor Castillo ${ }^{1}$ \\ ${ }^{1}$ Hospital International de Colombia and EMICON Research Group, Colombia \\ ${ }^{2}$ Universidad Manuela Beltrán, Bucaramanga, Colombia \\ ${ }^{3}$ University of Santander, Bucaramanga, Colombia
}

*Corresponding author: Mauricio Orozco Levi, International Hospital of Colombia, Kilometer 5 Autopista Piedecuesta, Floridablanca, Santander, Colombia.

To Cite This Article: Mauricio Orozco Levi, Diana Carolina Tiga Loza, Mabel Reyes Pulido, Alba Ramírez Sarmiento Victor Castillo. Frequency of Face and Mask Contacts Before and After Quarantine During COVID-19 Pandemics. Am J Biomed Sci \& Res. 2021 - 13(3). AJBSR.MS.ID.001865. DOI: 10.34297/AJBSR.2021.13.001865.

Received: 眥 June 16, 2021; Published: 跙 June 24, 2021

\begin{abstract}
Introduction: The World Health Organization released recommendations including social distancing, use of personal protective equipment, and respiratory hygiene to decrease the risk of coronavirus disease (COVID-19).

Objective: to estimate the number of potentially infectious self-contact in people located in concurred areas before and during the quarantine.

Methods: We conducted an observational study in Bucaramanga and nearby cities in Colombia, before and during the mandatory quarantine due to COVID-19 pandemic. Trained health professionals recorded the times that people touched their faces (nose, mouth, and eyes) or their masks with the hand or other fomites (cell phones, pens, etc.). Sex, apparent age, and observation time were also recorded.

Results: We observed 87 people at a hospital, a university, banks, and stores/wholesales, The mean observation time was 14.1 minutes (minimum 3 and maximum 66). A potential infectious self-contact occurred every 2.43 minutes. Before quarantine, the mean rate of hand-to-face contact was 30.4(9-88.4) times/hour and between fomites-to-face was 6.8(0-40) times/hour. During quarantine, the self-contact between handsmasks was 17.4(0-60) times/hour and between fomites-masks was 3.6(0-20) times/hour. We found a reduction in total contact rates after the quarantine $(\mathrm{p}=0.038)$, especially with fomites $(\mathrm{p}=0.027)$; women were more likely to self-contact (IRR:1.2, 95\% CI:1.09-1.32) also people between 25 and 34 years $(1.2,1.05-1.39)$. The places with the highest frequency of contact were the university $(1.39,1.20-1.60)$ and banks $(1.57,1.28-1.92)$.

Discussion: The recommendation for handwashing may not be sufficient on its own, because the frequency of contact may be greater than that of washing or the use of hand sanitizer.
\end{abstract}

Keywords: Coronavirus infections, Quarantine, Infection control, Disease transmission, Fomites, Behaviour, Personal protection

\section{Background}

On March 11, 2020, the World Health Organization (WHO) decreed this disease as a pandemic caused by the SARS-CoV-2, with more than 118,000 cases in 114 countries and 4,291 deaths at that time [1]. Some respiratory infections are partly the product of contacts of the hands with entry ports like the mouth, nose, and eyes. This contact is a relevant component in the chain of transmission in diseases such as rhinovirus and influenza [2,3]. The WHO's guidelines identify as the main source of the COVID-19 contagion inhaling the residual droplets generated by coughing or sneezing of people infected with respiratory symptoms [4]. Additionally, transmission can be caused by direct contact with infected people or with contaminated surfaces. As a secondary source, the generation of aerosols is a critical risk factor [5], especially in therapeutic clinical procedures such as endotracheal intubation, bronchoscopy, among others [4]. Therefore, the interventions promoted during the pandemic have been mainly handwashing, 
the use of Personal Protective Elements (PPE) such as face masks, gloves, goggles, and gowns, as well as surface disinfection and social distancing [6]. Available evidence on the effect of these measures in case and control studies, cohorts, and trials suggest the use of PPEs could result in reducing the spread of the SARS-CoV-2, but there exist inconsistent results and a low level of certainty. Independent interventions (only one intervention) such as hand washing alone do not show enough evidence of a positive effect on propagation [7].

Many countries declared mandatory quarantine as part of their containment and mitigation strategies aimed at older adults and children. Restriction of the mobility of the general population, as well as the obligatory use of masks, was also recommended with special attention to public transport users, symptomatic respiratory persons, and risk groups such as older adults and/or people with chronic or immune diseases [8,9]. These measures are not infallible, can generate a perception of false safety, and hide an inappropriate use of elements such as face masks. These elements appear to be frequently touched with hands after contact with potentially contaminated surfaces, or involuntary behaviours such as touching the face and its mucous membranes with hands or objects that may have been exposed to the virus. It was considered very relevant to evaluate the behaviour of people regarding the involuntary contact of their face with hands and fomites despite being currently sensitized to the high risk of COVID-19 transmission. Therefore, our objective was to estimate the number of contact of hands and fomites with the face and mask, in people located in health, educational, and commercial sectors before and after the quarantine was established.

\section{Methods}

\section{Design, Settings, and Participants}

We conducted an observational study in people over 15 years of age apparently, who were in areas prone to agglomeration in health, education, and commercial sectors. The investigation began 10 days after the first confirmed case report of COVID-19 in Colombia and we carried out two observation periods, before and after the establishment of quarantine and mandatory use of face masks. We choose for convenience a third-and fourth level of care hospital and a health section faculty from a university in the metropolitan area of Bucaramanga for pre-quarantine evaluation. We made duringquarantine observation in the same hospital in waiting rooms, and the waiting lines in some large-area stores/warehouses, and banks authorized for operation and public service by the national government.

\section{Variables}

We recorded the apparent age range and sex, location, number of contacts according to source (hands or fomites) and destinations (mouth, nose, and eyes or masks), and observation time, considering their possible combinations identifying as fomite to the cell phone, pencil/pen among other objects. We made a comparison between contact rates before and during quarantine, for this, only the contacts to the nose and mouth were counted prior-quarantine and compared with the contacts to the face mask during the quarantine.

\section{Data Source}

Trained health professionals choose one of every two people who entered the site and recorded the count of self-contact during the time the observed person remained at the site. They collected data in a paper form pre-validated by experts and were trained in standardizing the measurement of apparent age and sex using a personal reference according to the age range and sex, and developing strategies to avoid that participants felt observed and modified their behaviour, including not making direct eye contact, engaging in activities similar to those expected at assessment sites, pretending to use a cell phone, and temporarily suspending observation when the chosen person turned to see the evaluator.

\section{Statistical Methods}

We entered the collected data in Excel and analysed in STATA 13-SE. We described sociodemographic variables using measures of central tendency (mean and median) and dispersion (standard deviation and minimum-maximum values), frequencies and percentages; to obtain the contact rate per hour, the frequency of contacts was multiplied by 60 minutes and divided by the observation time. We used the Pearson correlation to evaluate the correlation between the different contact types. Due to the high variability of the data, we compared the median contact rates, between place, person, and time of observation variables using Kruskal Wallis and Wilcoxon-Signed Ranges tests. To compare rates ratio according to age, gender, and location and to adjusted rates of contact by these variables we used Poisson's regression model.

\section{Ethical Considerations}

This is a risk-free study according to Colombia's 1993 resolution 08430, we did not use the informed consent procedure because we consider the change in the behaviour in people who knows to be under observation, additionally, we recorded the frequency of self-contact hands/fomites-face anonymously, and no data were collected to identify each of the people observed beyond age and apparent sex.

\section{Results}

We observed 87 persons between March 16 and April 27, 2020. Fifty $(57.5 \%)$ observations were carried out during the second week after the start of the pandemic in Colombia when quarantine had not yet been declared, and $37(42.5 \%)$ two weeks after the establishment of the obligatory use of face masks during the generalized mandatory quarantine. 
Table 1 shows the characteristics of the observed people, time, and place, highlighting a similar distribution according to sex and age. We made most observations in a hospital (37.9\%) and the waiting lines in commercials places (34.5\%). A total of 1,230 minutes or 20.5 person-hours of observation were accumulated. The mean of contact rate between hands and face (eyes, nose, or mouth) was 30.4 times/hour. Contact with the mouth was the most frequently (14.7 times/hour); concerning the fomites, the cell phone contact presented a count of 4.7 times/hour (Table 2). Following the establishment of the quarantine, the face mask contact rate was 17.4 times/hour, and the fomites were 3.6 times/ hour. A substantial inter-individual variability was observed at all contact rates. An example of this is the contact rate of handsto-face that can range from zero contacts up to 88.4 contacts per hour (Table 2). No correlation was found between contact rates according to source and destination.

Table 1: Description of people's characteristics, time, and observation site - Bucaramanga Metropolitan Area, Colombia 2020.

\begin{tabular}{|c|c|c|}
\hline & $\mathrm{n}=87$ & $\%$ \\
\hline \multicolumn{3}{|c|}{ Observation Period } \\
\hline Before quarantine & 50 & 57.5 \\
\hline During quarantine & 37 & 42.5 \\
\hline \multicolumn{3}{|c|}{ Sex } \\
\hline Male & 43 & 49.4 \\
\hline Female & 44 & 50.6 \\
\hline \multicolumn{3}{|c|}{ Perceived Age } \\
\hline $15-24$ & 22 & 25.3 \\
\hline $25-34$ & 22 & 25.3 \\
\hline $35-49$ & 22 & 25.3 \\
\hline 50 or more & 21 & 24.4 \\
\hline \multicolumn{3}{|c|}{ Type of Institution } \\
\hline Hospital & 33 & 37.9 \\
\hline Store/warehouse & 26 & 29.9 \\
\hline University & 24 & 27.6 \\
\hline Bank & 4 & 4.6 \\
\hline \multicolumn{3}{|c|}{ Sites } \\
\hline Cafeteria & 12 & 13.8 \\
\hline Office/service & 4 & 4.6 \\
\hline Waiting lines & 30 & 34.9 \\
\hline Common college areas & 17 & 19.5 \\
\hline Hospital waiting room & 24 & 27.6 \\
\hline
\end{tabular}

Table 2: Contact rate (hourly counts) raw and adjusted, depending on the type of contact and source, before and after quarantine - Bucaramanga Metropolitan Area, Colombia 2020.

\begin{tabular}{|c|c|c|c|c|c|}
\hline \multirow[b]{2}{*}{ Type of Contact } & \multirow[b]{2}{*}{$\mathbf{n}$} & \multicolumn{2}{|c|}{ Raw Contact Rate } & \multicolumn{2}{|c|}{ Adjusted Contact Rate* } \\
\hline & & Mean (St. Dev.) & Mediana (min-max) & Mean (St. Dev.) & Mediana (min-max) \\
\hline \multicolumn{6}{|l|}{ Before Quarantine } \\
\hline Hand-Mouth & 50 & $14.7(12.5)$ & $12.6(0-56.8)$ & $16.7(6.4)$ & $16(6.1-32.2)$ \\
\hline Hand-Nose & 50 & $6.6(9.2)$ & $3.1(0-40)$ & $7.6(3.6)$ & $7.1(2.3-15)$ \\
\hline Hand-Eyes & 51 & $9.1(8.8)$ & $6.6(0-30)$ & $7.6(7.2)$ & $5(0.7-50)$ \\
\hline Total Hands-Face & 50 & $30.4(16.7)$ & 28. $1(9-88.4)$ & $29.3(5.9)$ & $29.7(20.5-42)$ \\
\hline Cell-Face & 50 & $4.7(6.0)$ & $3.5(0-30$ & $4.7(3.1)$ & $4(1-13.6)$ \\
\hline Pen-Face & 50 & $1.5(6.6)$ & $0(0-40)$ & $2.4(6.1)$ & $0(0-24)$ \\
\hline Other Fomites-Face & 50 & $0.6(2.1)$ & $0(0-12)$ & $0.6(0.9)$ & $0.1(0-4.2)$ \\
\hline Total Fomites-Face & 50 & $6.8(8.2)$ & $5.3(0-40)$ & $7.9(3.2)$ & $6.5(3.9-13.9)$ \\
\hline \multicolumn{6}{|l|}{ After Quarantine } \\
\hline Hand-Face Mask & 37 & $17.4(17.2)$ & $16.1(0-60)$ & $30.9(27.8)$ & $18.1(8.2-90.3)$ \\
\hline
\end{tabular}




\begin{tabular}{|c|c|c|c|c|c|}
\hline Fomites-Face Mask & 33 & $3.6(5.9)$ & $0(0-20)$ & $4(3)$ & $4(0.6-9)$ \\
\hline Contact with Hands & 87 & $24.9(20)$ & $22.5(0-88.4)$ & $24.9(8.2)$ & $24.1(10.6-40.8)$ \\
\hline Contact with Fomites & 87 & $5.3(7.4)$ & $2.5(0-40)$ & $5.3(2)$ & $5.7(0-9.4)$ \\
\hline Total contacts & 87 & $24.5(18.1)$ & $20(0-85.2)$ & $24,6(5.6)$ & $24.7(13.8-35.9)$ \\
\hline
\end{tabular}

We found significant differences concerning apparent age. People between 15 to 24 years had a lower contact rate with the face $(p=0.0382$ ) (Figure 1a), while those between an age of 35 and 49 years exhibited the highest rate of hand-mouth contact $(p=0.0379)$ (Figure 1b). No differences were evident by sex when the origin of the contact was the hands (Figure 2a), but women had a higher rate of contact with the cell phone $(p=0.019)$ and with the fomites to the face $(p=0.0153)$ (Figure $2 b)$. Also, the hand contact was lower in the store/warehouse checkout lines $(p=0.0122)$, as well as in the bank waiting lines, the contact rate per fomites was lower $(\mathrm{p}=0.032$ ) (Figure 3). Also, during-quarantine we found a reduction in total contact rate $(p=0.038)$ and by fomites $(p=0.027)$ (Figure
4). We adjusted the contact rates, by differences found according to age, gender, and place, (Table 2) and estimated that a potentially infective contact occurs every 2.43 minutes (1.7-4.3) and if the contact does not vary during 12-hour wakefulness period, can be occurred a mean of 297 contacts/day. Through Poisson's regression model we estimated that the contact rate is $20 \%$ higher in women compared to men, $20 \%$ higher in those who appear to be between 25 and 34 years old, and 22\% lower in those who are more than 50 years old compared to the youngest group; as well as 18\% higher in hospitals, $39 \%$ in university and $57 \%$ in banks compared to contact in-store/warehouse (Table 3).

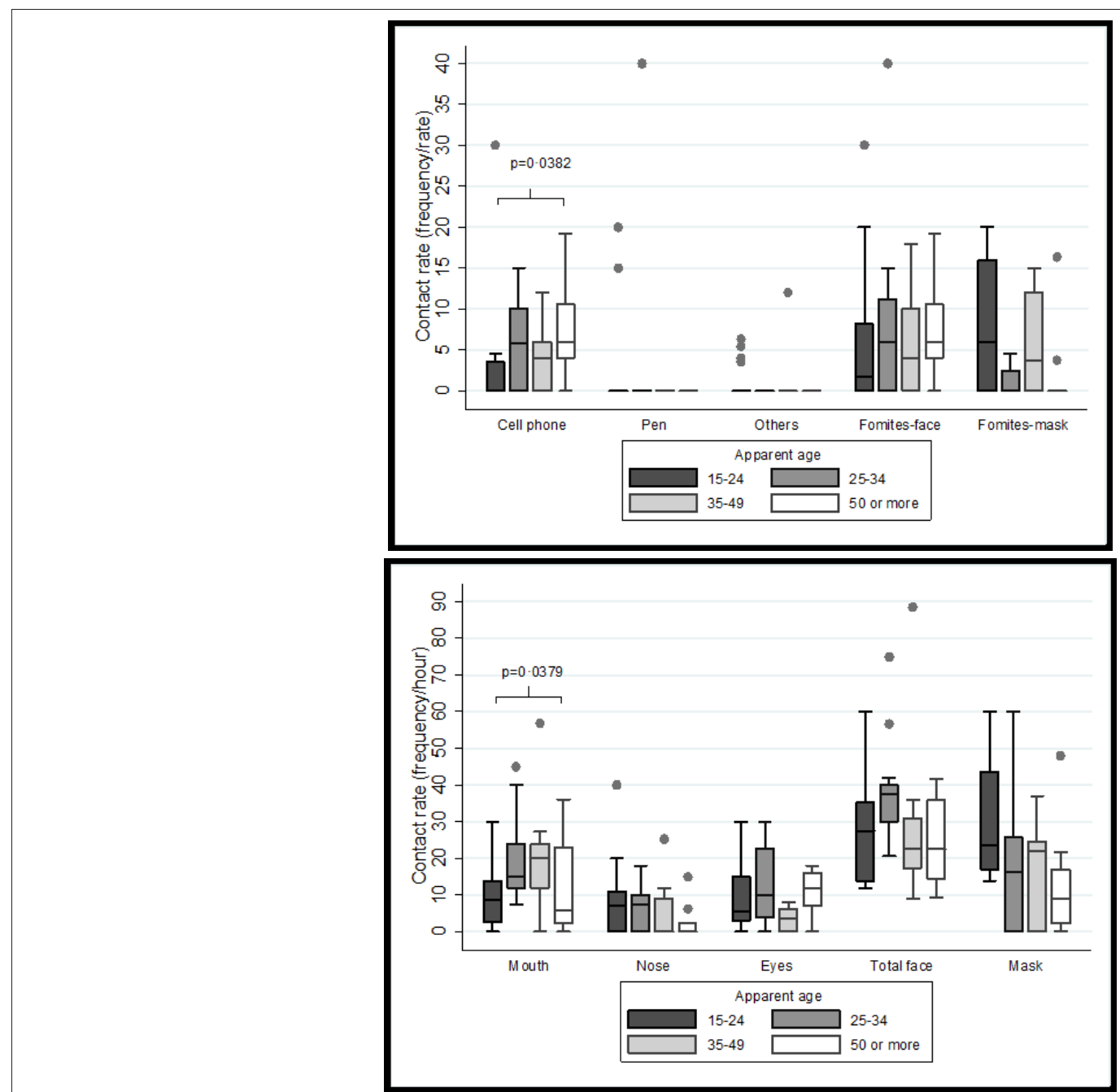

Figure 1: Distribution of hourly contact rates according to apparent age group - Bucaramanga Metropolitan Area, Colombia 2020.

a: Contact rates by age and destination whose contact source were hands.

b: Contact rates by age whose contact source was the fomites. 


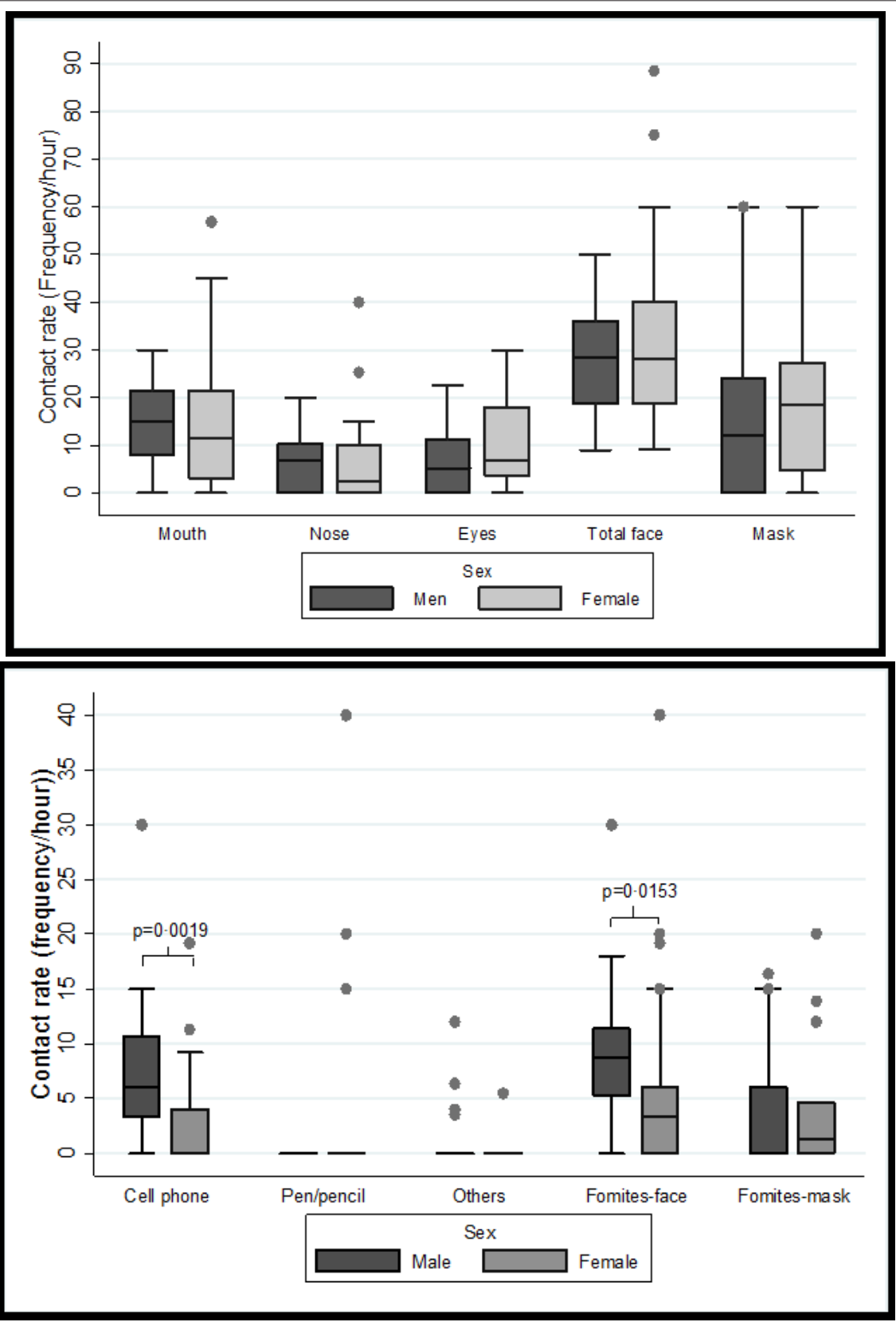

Figure 2: Distribution of hourly contact rates according to apparent sex group - Bucaramanga Metropolitan Area, Colombia 2020. a: Contact rates by sex and destination whose contact source were hands.

b: Contact rates by sex whose contact source was the fomites. 


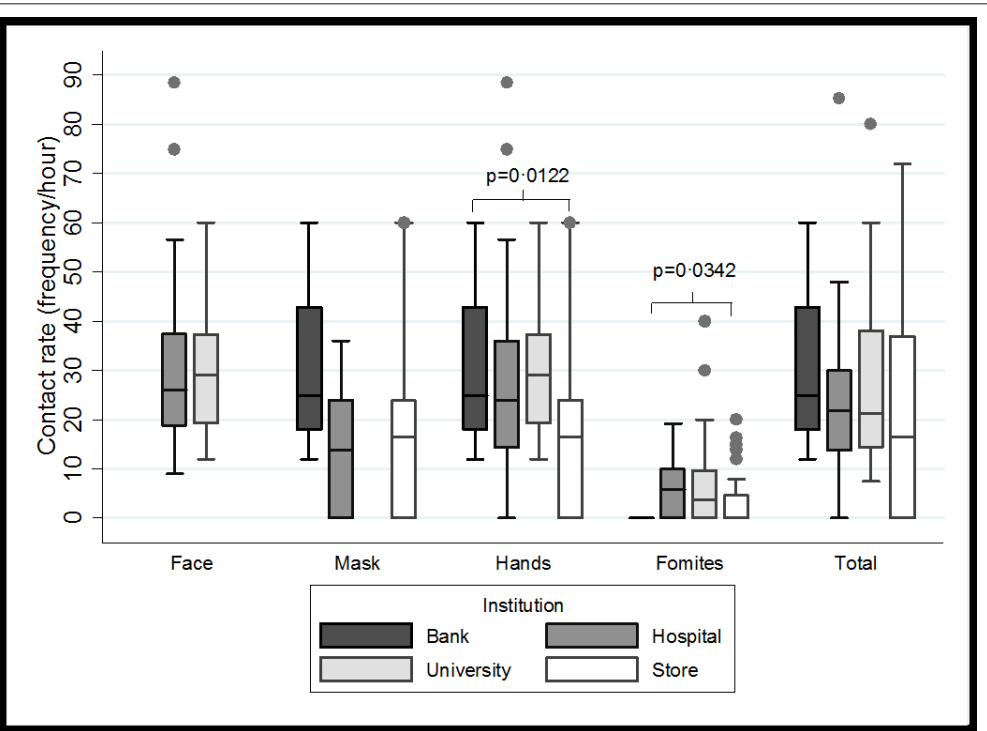

Figure 3: Distribution of contact rates by origin and destination according to the place of observation - Bucaramanga Metropolitan Area, Colombia 2020.

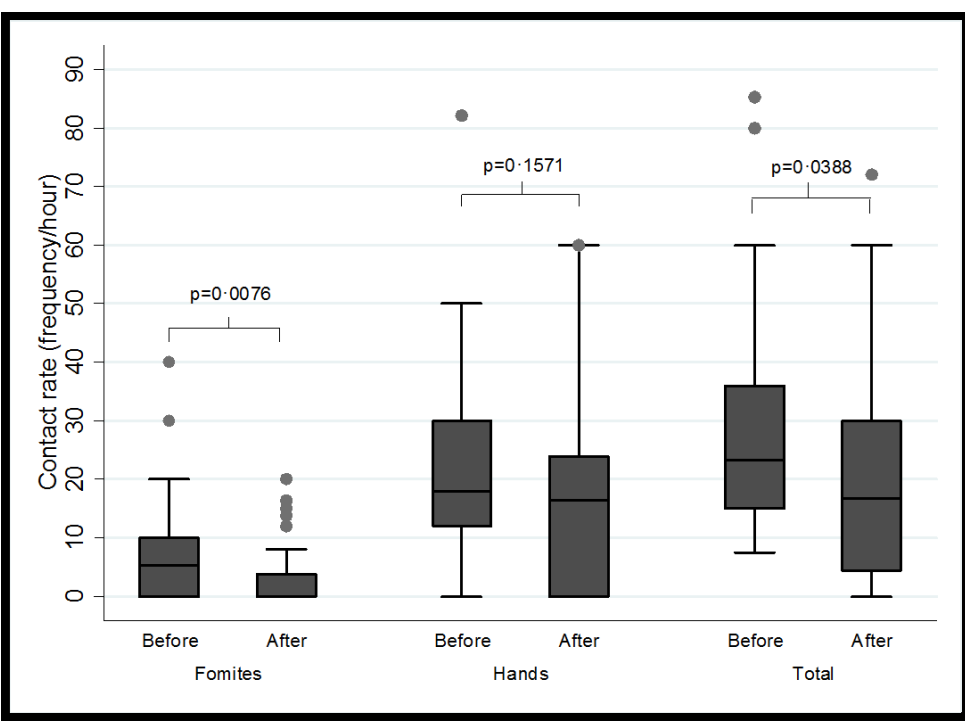

Figure 4: Distribution of contact rates to mouth/nose or face mask, depending on origin, before and after the generalized mandatory quarantine establishment - Bucaramanga Metropolitan Area, Colombia 2020.

Table 3: Rate ratio of contact by sex, apparent age, and place - Bucaramanga Metropolitan Area, Colombia 2020.

\begin{tabular}{|c|c|c|c|c|}
\hline & \multirow{2}{*}{ Rate ratio } & \multirow{2}{*}{ p-value } & \multicolumn{2}{|c|}{$95 \%$ confidence interval } \\
\hline & & & Upper & Lower \\
\hline \multicolumn{5}{|c|}{ Sex } \\
\hline Male & Reference & & & \\
\hline Female & 1.2 & $<0.001$ & 1.09 & 1.32 \\
\hline \multicolumn{5}{|c|}{ Apparent Age } \\
\hline $15-24$ years old & Reference & & & \\
\hline $25-34$ years old & 1.2 & 0.008 & 1.05 & 1.39 \\
\hline $35-49$ years old & 1.18 & 0.065 & 0.99 & 1.4 \\
\hline 50 or more & 0.78 & 0.004 & 0.65 & 0.92 \\
\hline
\end{tabular}




\begin{tabular}{|c|c|c|c|c|}
\hline \multicolumn{5}{|c|}{ Institution } \\
\hline Store/wholesale & Reference & & & \\
\hline Hospital & 1.18 & 0,004 & 1.05 & 1.32 \\
\hline University & 1.39 & $<0.001$ & 1.28 & 1.6 \\
\hline Bank & 1.57 & $<0.001$ & 1.92 \\
\hline
\end{tabular}

\section{Discussion}

The present study demonstrated that the frequency of potentially infectious self-contact is very high in pandemic times. We found the contact rate of hands-face was 29.3 times/hour, like that reported by Hendley et al., between 29 and 33 times/hour [2], although other studies have reported lower rates 15.7 - 23 times/ hour $[10,11]$. Such differences could happen due to the type of population studied, but all these studies emphasize that the contacts are tenths of times per hour. Our study was conducted in different places that include diversity in the people studied, while Kwok, et al. [11] carried out observations in students only. On the other hand, the time of observation can also influence the differences between the studies, our study observed 20.5 hours-person, lower compared to other studies with 30 and 89 hours-person observation time $[2,10]$. Another possible reason for discrepancies between rates could be that in our study, observations were made in naturally frequented places, instead the study of Nicas et al. [10], participants were in the absence of social contact, in a controlled environment, and recorded by a camera. In the present study, most registered contacts were hand-to-mouth with a rate of 17 times/ hour. Secondly hand-to-nose (median=7.1 times/hour) and thirdly hand-to-eyes (median=5 times/hour). This trend is like that found by Kwok et al., whereas far as the touches to mucous membranes were observed, $36 \%$ involved the mouth, 31\% involved the nose, and $27 \%$ the eyes [11].

For the control, prevention, and containment of SARS-CoV-2 infection, WHO has disseminated strategies to wash hands frequently, respiratory hygiene measures such as coughing or sneezing covering the face with the elbow or handkerchief, maintaining social distancing, and finally, avoid touching the eyes, nose, and mouth with hands to prevent the self-inoculation [6]. In this study, we observed that despite the measures of use of mandatory face masks and social distancing, people often tend to touch their face or face masks frequently.

Several studies have been conducted to assess the effect of handwashing on the rate of airway infections with positive results, in different population contexts such as the university, geriatric homes, and military barracks [12-14]. Meta-analysis studies report conflicting results ranging from an effect on decreased respiratory symptoms and diseases such as influenza [15], to a non-significant effect on laboratory-confirmed influenza reduction $(\mathrm{RR}=0.82$; 95\%IC 0.66-1.02; $p=0.07$ ] [16], possibly because the frequency of handwashing is less than the frequency of potentially contaminating self-contact. We found that the frequency of self-contact was approximately 300 times/ day and Nicas et al. [10], observed an average of auto-contact of 15.7 times per hour; nevertheless, it is considered a high frequency of handwashing 10 times or more per day [17]. This indicates that the frequency of contaminating selfcontact is higher than the frequency of handwashing. Also, this reinforces the need for an effective hand-washing protocol as often as possible, accompanied by barrier and disinfection measures, with attention in places with greater potential for contagion such as hospitals, universities, and commercial establishments. Likewise, in a meta-analysis for influenza, reports that there is no evidence of an important effect on transmission when the intervention evaluated were handwashing with the use of a mask $(R R=1.05$, 95\%CI 0.86 1.27 ; $\mathrm{I} 2=57 \%, \mathrm{p}=0.65)$, without a mask $(\mathrm{RR}=0.78,95 \% \mathrm{CI} 0.51$ $1.20 ; \mathrm{I} 2=30 \%, \mathrm{p}=0.25$ ) or cleaning procedures of contaminated objects and surfaces [18]. On the other hand, Chu and others, [19] found an effect in the decrease of viral transmission due to distance (OR 0.18, 95\% CI 0.09 to 0.38), use of masks (OR 0.15, 95\% CI 0.07 to 0.34 ), and eye protection (OR $0.22,95 \%$ CI 0.12 to 0.39 ).

From a neurobiological point of view, face touching is a frequent behaviour in humans and all primates and, could be related to the tendency of humans to self-sniff. Observing primates and humans for 20 minutes, found an equivalent frequency of contacts between them with variations depending on the activity being carried out, whether it is known that the participants are observed or not [20]. It is also presumed that face touching as a way of self-smelling is largely an unconscious act, for instance, a study in 400 participants, found $94 \%$ of them admitted having smelled of themselves [21].

On the other hand, the behaviour towards COVID-19 may be influenced by fear, social norms, and culture [22]. Fear can lead to people making changes in their behaviour when they feel capable of facing the threat, or on the contrary, it produces defensive reactions when they feel incapable to act [23]. A possible explanation for the high rate of self-contact is a low perceived self-efficacy for lack of control of a movement such as self-contact of the face, which is generally carried out unconsciously. Similarly, social norms influence the behaviour of people, who can be highly reactive to the behaviours of others; however, in some cases, there may be an underestimation of favourable behaviours such as handwashing or the use of masks [24]; the same could be happening with selfcontact, having a diminished perception of the danger that such a habitual act represents. Also, the population of Latin American 
origin is characterized by greater body expressiveness and affective contact [25], which could partly explain the high rate of contacts that we found in a Latin American country like Colombia, despite the similar rate found in the USA [2]. Nicas and col. [10] found that those who touch their lips often tend to touch their nose and eyes more while we did not observe these correlations.

It should be noted that hands are a vehicle for the transfer of respiratory pathogens in activities of daily living. In a study that evaluated rhinovirus contamination in hotel rooms inhabited by sick people using RT-PCR to surfaces such as the light switch, tv remote control, taps, telephones, and door handles, finding rhinovirus positive on $35 \%$ of sites. Additionally, contamination of hands was evaluated, finding positives $60 \%$ during exposure and $33 \%$ after 18 hours from contact [26]. On the contrary, we think the self-contact may be less in hospitals and supermarket lines, possibly because people perceive a higher probability of contagion in these places. Self-contact in banks was less, probably due to the security restriction of cell phone use. Also, we found that younger people have less contact with their cell phones, possibly because the interaction with screens is greater than with voice [27].

This study is one of the few that measures the frequency of contacts and is the first approach in Latin America. It is a study carried out in real-life scenarios evidencing the high frequency of such involuntary acts in contrast to WHO's recommendations to avoid such contact. Adherence to these guidelines is low and difficult to sustain, reflecting the necessity to promote barrier strategies that prevent involuntary contact and empowerment in terms of handwashing as often as possible. The limitation identified is that being an observational study of social behaviour, the amount of information that the observed persons had previously received on COVID-19 was not homogenized. It is assumed that they have been widely sensitized but the degree of internalization and understanding of the information in this regard is not known. People's behaviour may be influenced by context, which decreased self-contact in locations with the most probability of contagion, such as hospitals and supermarkets. The reproducibility of the observations is not known; this requires an evaluation by at least two observers to the same subject, and training of the evaluators was carried out to reduce intra-observer variability.

The adherence to the recommendation of avoiding the face and mask contacts is very low. Despite being in a period of a pandemic due to coronavirus, we confirm that number of contacts of the hands with parts of the face (eyes, nose, mouth), the number of contacts with fomites (cell phones, pens, or pencils) is very high both in university students and in health professionals and users of hospitals, banks, and shops. This indicates that the advice on contact precautions is insufficient, possibly due to the reflexive and involuntary nature of these actions. We cannot continue to place so many expectations on the abolition of facial contacts and we must investigate additional ingenious ways (possibly sonorous electronic devices) that allow reducing the risk of self-infection or inter-personal transmission by SARS-CoV-2 on contact with the face.

\section{Declaration of Conflicting Interests}

The authors declared no conflicts of interest in this work.

\section{Funding}

Partial support by MINCIENCIAS (Grant CODE:656677758319) and Hospital Internacional de Colombia.

\section{References}

1. (2020) WHO Director-General's opening remarks at the media briefing on COVID-19 - 11 March 2020. World Health Organization Speeches of Director-General WHO.

2. Hendley JO, Wenzel RP, Gwaltney JMJ (1973) Transmission of rhinovirus colds by self-inoculation. $\mathrm{N}$ Engl J Med 288(26): 1361-1364.

3. Atkinson MP, Wein LM (2008) Quantifying the routes of transmission for pandemic influenza. Bull Math Biol 70(3): 820-867.

4. WHO (2020) Modes of transmission of virus causing COVID-19: implications for IPC precaution recommendations. Scientific brief pp.1.

5. van Doremalen N, Morris DH, Holbrook MG, Gamble A, Williamson BN, et al. (2020) Aerosol and Surface Stability of SARS-CoV-2 as compared with SARS-CoV-1. New Engl J Med 382: 1564-1567.

6. WHO (2020) Coronavirus disease (COVID-19) advice for the public.

7. Burch J, Bunt C (2020) Can physical interventions help reduce the spread of respiratory viruses? Cochrane Clin Answers.

8. (2020) The use of face masks is mandatory in the public transport system. Press Bulletin No 125, Ministry of Health and Social Protection.

9. Anderson RM, Heesterbeek H, Klinkenberg D, Hollingsworth TD (2020) How will country-based mitigation measures influence the course of the COVID-19 epidemic? Lancet 395(10228): 931-934.

10. Nicas M, Best D (2008) A study quantifying the hand-to-face contact rate and its potential application to predicting respiratory tract infection. J Occup Environ Hyg 5(6): 347-352.

11. Kwok YLA, Gralton J, McLaws M (2015) Face touching: A frequent habit that has implications for hand hygiene. Am J Infect Control 43(2): 112114 .

12. White C, Kolble R, Carlson R, Lipson N, Dolan M, et al. (2003) The effect of hand hygiene on illness rate among students in university residence halls. Am J Infect Control 31(6): 364-370.

13. Falsey AR, Criddle MM, Kolassa JE, McCann RM, Brower CA, et al. (1999) Evaluation of a Handwashing Intervention to Reduce Respiratory Illness Rates in Senior Day-Care Centers. Infect Control Hosp Epidemiol 20(03): 200-202.

14. Ryan M, Christian R, Wohlrabe J (2001) Handwashing and respiratory illness among young adults in military training. Am J Prev Med 21(2): 79-83.

15. Willmott M, Nicholson A, Busse H, Macarthur GJ, Brookes S, et al. (2016) Effectiveness of hand hygiene interventions in reducing illness absence among children in educational settings: A systematic review and metaanalysis. Arch Dis Child 101(1): 42-50.

16. Wong VWY, Cowling BJ, Aiello AE (2014) Hand hygiene and risk of influenza virus infections in the community: A systematic review and meta-analysis. Epidemiol Infect 142(5): 922-932. 
17. Beale S, Johnson AM, Zambon M, Hayward AC, Fragaszy EB, et al. (20202) Hand and Respiratory Hygiene Practices and the Risk and Transmission of Human Coronavirus Infections in a UK Community Cohort. Wellcome Open Res 2020 5(98): 1-9.

18. Xiao J, Shiu EYC, Gao H, Wong JY, Fong MW, etal.(2020) Nonpharmaceutical Measures for Pandemic Influenza in Nonhealthcare Setting-Personal Protective and Environmental Measures. Emerg Infect Dis 26(5): $967-$ 975.

19. Chu DK, Akl EA, Duda S, Solo K, Yaacoub S, et al. (2020) Physical distancing, face masks, and eye protection to prevent person-to-person transmission of SARS-CoV-2 and COVID-19: a systematic review and meta-analysis. Lancet 395(10242): 1973-1987.

20. Dimond S, Harries R (1984) Face touching in monkeys, apes and man: Evolutionary origins and cerebral asymmetry. Neuropsychologia 22(2): 227-233.

21. Perl O, Mishor E, Ravia A, Ravreby I, Sobel N (2020) Are humans constantly but subconsciously smelling themselves? Philos Trans R Soc B Biol Sci 375(1800): 20190372.
22. Van Bavel JJ, Baicker K, Boggio PS, Capraro V, Cichocka A, et al. (2020) Using social and behavioural science to support COVID-19 pandemic response. Nat Hum Behav 4(5): 460-471.

23. Witte K, Allen M (2000) A meta-analysis of fear appeals: Implications for effective public health campaigns. Heal Educ Behav 27(5): 591-615.

24. Dickie R, Rasmussen S, Cain R, Williams L, MacKay W (2018) The effects of perceived social norms on handwashing behaviour in students. Psychol Health Med 23(2): 154-159.

25. Burleson MH, Roberts NA, Coon DW, Soto JA (2019) Perceived cultural acceptability and comfort with affectionate touch: Differences between Mexican Americans and European Americans. J Soc Pers Relat 36(3): 1000-1022.

26. Winther B, Mccue K, Ashe K, Rubino JR, Hendley JO (2007) Environmental contamination with rhinovirus and transfer to fingers of healthy individuals by daily life activity. J Med Virol 79(10): 1606-1610.

27. Langer CE, de Llobet P, Dalmau A, Wiart J, Goedhart G, et al. (2017) Patterns of cellular phone use among young people in 12 countries: Implications for RF exposure. Environ Int 107: 65-74. 